Editorial

\title{
Asthma-COPD overlap syndrome: the different faces or a new entity?
}

\section{Editorial}

Several epidemiologic studies revealed that half or more of older patients with obstructive airway disease may have overlapping diagnosed of asthma and chronic obstructive pulmonary disease (COPD) (overlap syndrome). Overlap syndrome can develop when there is incomplete lung growth and accelerated decline in lung function. The diagnosis of asthma-chronic obstructive pulmonary disease overlap syndrome (ACOS) may be difficult due to the lack of specific biomarkers, whereas asthma and COPD have clear differences. ACOS patients are more likely to present 2 or more exacerbations per year to contribute to the more worsening of respiratory symptoms, especially dyspnea and wheezing, reduction of physical activity, and worsening the quality of life, than the COPD patients. ACOS patients have better response to inhaled corticosteroids (ICS) compared to those with COPD. Some investigators defined ACOS as asthma with partially reversible airflow obstruction, with reduced carbon monoxide diffusing capacity (DLCO) to less than $80 \%$ predicted or with or without emphysema, and COPD with emphysema accompanied by partially reversible or reversible airflow obstruction, with reduced DLCO or with or without environmental allergies. COPD patients (post-bronchodilator FEV1/FVC $<0.7$ and more than 10 pack-years of smoking) with personal history of asthma before the age of 40, absence of pulmonary emphysema, and with the additionally normal DLCO was also defined as ACOS. Nevertheless, ACOS has been conclusively defined as increasing variability of airflow in association with incompletely reversible airflow obstruction. Importantly, an asthmatic patient with chronic non-fully reversible airflow obstruction is not necessarily ACOS. While in COPD patients, there is neutrophillic inflammation and driven by $\mathrm{CD} 8+$ cells, there is mainly eosinophilic and mediated by CD4+ T lymphocytes in COPD patients with ACOS. The most important diagnostic feature of COPD patients with ACOS is possibly the presence of sputum eosinophilia. The prevalence of ACOS varies from $3.9 \%$ to $21.2 \%$ among COPD patients. Reduction in eosinophilic concentrations is the novel treatment targets. Anti-interleukin 5 (benralizumab)-treated patients with the highest eosinophilic count showed a reduction of exacerbation rates and improvements in FEV1, except the reduction of exacerbations in COPD patients with peripheral eosinophilia. Whether COPD patients with eosinophilia at
Volume 3 Issue 5 - 2016

\author{
Attapon Cheepsattayakorn, ${ }^{1,2,3}$ Ruangrong \\ Cheepsattayakorn ${ }^{4}$ \\ 'Editor-in-Chief, Journal of Lung, Pulmonary and Respiratory \\ Research, USA \\ ${ }^{2}$ IOth Zonal Tuberculosis and Chest Disease Center, Chiang Mai, \\ Thailand \\ ${ }^{3} 5$ th Office of Disease Prevention and Control, Ratchaburi, \\ Department of Disease Control, Ministry of Public Health, \\ Thailand \\ ${ }^{4}$ Department of Pathology, Faculty of Medicine, Chiang Mai \\ University, Chiang Mai,Thailand
}

Correspondence: Attapon Cheepsattayakorn, I0th Zonal Tuberculosis and Chest Disease Center, 143 Sridornchai Road Changklan Muang Chiang Mai 50100 Thailand,Tel 6653 I40767, 6653 276364, Fax 6653 140773, 6653 273590,

Email Attapon1958@gmail.com, attaponche@yahoo.com

Received: November 07, 2016 | Published: November 17, 2016

adult age can develop ACOS over a lifespan is of interest. ICS may improve asthma remodeling and affect asthma outcomes, whereas they reduce asthmatic exacerbations. A longitudinal prospective population study, such as deoxyribonucleic acid (DNA) and nasal epithelial cells for (epi)genetic assessments that follows anyone with respiratory symptoms, involving the whole spectrum of future disease would be an ideal study. In conclusions, there are three characteristic features in ACOS patients: 1) increased response to ICS compared to the patients with COPD alone, 2) increased reversibility of airflow, and 3 ) enhanced bronchial and systemic eosinophilic inflammation.

\section{Acknowledgements}

None.

\section{Conflict of interest}

The author declares no conflict of interest. 